\title{
Ethical Issues in Pharmacy Practice Research: An Introductory Guide
}

Peter Loewen

\section{INTRODUCTION}

$\mathrm{B}$ $y$ its nature, pharmacy practice and pharmacotherapy research, referred to here as "pharmacy practice research", usually involves humans as research participants in one way or another. Consequently, researchers must understand the implications of their work from the viewpoint of protecting the rights and interests of participants. Although there are dozens of guidelines and regulatory documents governing research ethics worldwide, Canadian researchers can learn what these interests and rights are by reading the Tri-Council Policy Statement on Ethical Conduct for Research Involving Humans (TCPS). ${ }^{1}$ The TCPS is the main governing document for research ethics in Canada. As research and societal values evolve, so too does the TCPS and its interpretations. Many universities and hospitals require completion of a TCPS tutorial ${ }^{2}$ as a condition of conducting research involving humans. The information in the tutorial is essential knowledge, and research participants deserve to interact only with ethically astute researchers, regardless of the level of risk involved. Using people as research participants is a privilege, and the public's trust in researchers is fragile and must be carefully nurtured by everyone involved.

Pharmacy practice researchers frequently encounter issues that require more specific advice than that offered by the TCPS. This article, while no substitute for reading the TCPS, advises on some key issues faced by pharmacy practice researchers. The time invested in reading this short article may yield a solid return in terms of time and frustration saved.

As a result of numerous egregious violations of the rights and interests of research participants, ${ }^{3-5}$ the privilege of using humans for research is granted only in the context of a regulated system of research ethics boards (REBs) and sometimes other levels of oversight, depending on the type of research. Although this system has probably prevented many tragic misadventures of the types encountered in the past, the changing nature of research continually reveals new issues and threats to participants' interests, keeping researchers and REBs busy adapting.
Salient examples include privacy threats from linkage of large-scale databases, changing societal privacy expectations, and personal and family implications of genomic research.

Researchers are required to obtain a certificate of approval to conduct research from one of the following types of REBs: university-based (covering on-campus research and frequently university-affiliated hospitals), hospital-based (covering only a particular hospital or family of hospitals, as in a specific health region), community (covering research not under the auspices of a hospital or health authority, such as private clinic-based research or community pharmacy research), or private (for-profit REBs that cover any research not covered by the other types of REB). All of these REBs conform to the TCPS requirements, but each has policies and expectations shaped by local history and culture. For example, the REB for a Catholic hospital may impose prohibitions on certain kinds of research (e.g., research involving abortion).

The Canadian and US systems of research ethics oversight are different. Whereas Canada relies primarily on giving guidance to researchers via the TCPS and expects REBs to uphold and interpret that guidance, the United States uses a federal legislative and regulatory approach. The US Food and Drug Administration (FDA) and the US Department of Health and Human Services operationalize federal laws and create regulations that all researchers must follow and that institutional review boards (analogous to REBs in Canada), which must be federally certified, must uphold. For example, the US Code of Federal Regulations - Title 21 contains dozens of specific rules about what must be included in a research consent form. ${ }^{6}$ Inspections, audits, and investigations of researchers and institutional review boards are sometimes performed by the federal Office for Human Research Protections. This means that Canadian REBs must maintain certification under the US system to be eligible to approve research sponsored by the National Institutes of Health or US-based, FDA-regulated pharmaceutical companies. I recently had the experience of chairing the first non-US REB to undergo auditing by the Office for Human Research Protections, and 
the process was certainly rigorous. The FDA maintains a comprehensive "frequently asked questions" (FAQ) page for researchers and institutional review boards about all these issues. $^{\text {? }}$

Researchers are sometimes required to obtain approval from more than one REB. A university-affiliated researcher doing research in a jurisdiction outside that covered by his or her university's REB is the most common scenario. Multisite projects represent another. Inter-REB cooperation or even reciprocation agreements sometimes exist, and a call to one of the REBs is advised before assuming that duplicate applications are required. If not, plan for extra time and work to satisfy multiple REBs with different application forms and expectations about, for example, consent forms. A contract research organization coordinating a single study at, say, 50 sites may be dealing with 50 different REBs and tracking 50 different versions of the consent form. In Canada, some early efforts to harmonize the REB approval process across sites are under way for large studies with many sites funded by the Canadian Institutes of Health Research. ${ }^{8}$ There are some provincial efforts to do this as well.9 In British Columbia, for example, the six REBs overseeing clinical research in the health authorities affiliated with the University of British Columbia regularly perform a harmonized review of studies being conducted at multiple sites spanning the REBs.

\section{WHAT ARE REBS LOOKING FOR?}

REB approval can be obtained more quickly by understanding the aspects of a research project in which the REB is most interested. REBs are very sensitive to the research protocol and associated documents (e.g., the consent form, assent form, interview scripts) being complete, coherent, and well thought-out. No REB wants to approve a research proposal that appears to be coming from a researcher who is not conscientious, thorough, qualified, properly resourced, and well organized. Regardless of the risk to participants, the research itself must make sense and be feasible. For example, REBs expect research questions to be clearly formulated and also expect the study design to address all of the research questions. REBs are frequently criticized for critiquing methodology as being unrelated to ethics. In fact, most REBs contend that research that cannot answer the questions posed is inherently unethical because it exploits participants to no useful end, diverts resources, and undermines public trust in the research process. Hence, ethical review almost always involves some level of scientific (sometimes called "scholarly") review, so the application for REB approval should be composed accordingly. Of course, all of the local requirements for application and study documents must be met. For studies comparing interventions, the REB must be satisfied that there is clinical equipoise, namely that there is genuine professional or scientific uncertainty about which intervention is better. The burden of equipoise escalates as risk increases. For example, an educational intervention for patients with chronic obstructive pulmonary disease compared with usual care for these patients might be held to a lower standard of equipoise than a new biologic being compared with standard care for sepsis. REBs pay a lot of attention to risk level (minimal or not). This is the primary decision point for many REBs when choosing fullboard versus expedited review streams.

Most REBs offer "expedited review" for projects involving minimal risk. Other conditions frequently apply (e.g., no collection of tissue, no creation of a registry, no use of radiation, no stem cells, no genetic research). This type of review bypasses the full REB meeting review through delegation of responsibility to a subset of members, sometimes just the chair.

There is some controversy about interpretations of minimal risk, but the TCPS defines projects involving minimal risk as "research in which the probability and magnitude of possible harms implied by participation in the research is no greater than those encountered by participants in those aspects of their everyday life that relate to the research." ${ }^{1}$ REBs take a proportional approach to ethics review, meaning that the intensity of review and oversight is proportional to the amount of risk. Pharmacy practice researchers should think carefully about whether their research carries minimal risk or not, and plan the REB application and consent documents accordingly. The final main focus of REB considerations is disclosure to research participants. Have all possible relevant implications of participation been disclosed? What about the purpose of the research? Every research-related procedure? All the risks? All the privacy implications? And, most importantly, does everything in the consent form align with what the protocol describes?

\section{WHAT OTHER KINDS OF REVIEW MIGHT MY RESEARCH REQUIRE?}

Nearly all hospitals require some sort of administrative approval before research can commence. This step focuses on the resource impacts of the research, rather than its ethical aspects. To confuse matters, many hospitals combine the ethics and administrative approval processes into one application that is handled by one office. In exchange for less paperwork, this combined process makes it hard for some researchers to distinguish ethical issues from administrative ones. Consider also whether your department has its own scientific review process that must be passed before the protocol is submitted to the REB. Additionally, trial registration (usually with clinicaltrials. gov) is required for "any research project that prospectively assigns people or a group of people to an intervention, with or without concurrent comparison or control groups, to study the causeand-effect relationship between a health-related intervention and a health outcome." ${ }^{10}$ Finally, research involving any drug 
being used outside its labelled indication must be approved by Health Canada via a Clinical Trial Application. ${ }^{11}$ It is wise to confirm this requirement with Health Canada before commencing the application process, because it is daunting.

\section{WHAT ARE THE COMMON ETHICAL ISSUES AFFECTING PHARMACY PRACTICE RESEARCHERS?}

\section{Need for Ethics Review}

Pharmacy practice researchers commonly ask, "Does my research require REB review?” Most REBs have an FAQ page to help in answering this question. The answer hinges on whether the project meets the TCPS definition of research ("an undertaking intended to extend knowledge through a disciplined inquiry or systematic investigation" ${ }^{1}$ ). If not, REB review is usually not required, although researchers should obtain some confirmation from the REB that this is the case. This definition is sometimes difficult for researchers and REBs to interpret, so requirements vary widely across institutions. Projects not requiring REB approval usually fall into the category of quality assurance/quality improvement research. Contrary to popular belief, research in this category is not excluded from publication. Some REBs will, upon request, give researchers a letter stating that they consider the research exempt from ethics review.

\section{Consent}

The second most frequently asked question is, "Do I have to get consent from participants?" If any direct contact with participants is involved, the answer is yes. If not, and the research involves only minimal risk, the main question is whether a waiver of consent under TCPS article 3.7 can be granted. There are 5 criteria that must be satisfied, although one of these, article 3.7(e), will probably be eliminated in an upcoming revision of the guideline. ${ }^{12}$ Most REBs have an essentially automatic approach to granting such waivers when only retrospective chart review is involved. However, pharmacy practice researchers frequently wish to have consent requirements waived for prospective observational studies. This is harder to justify under the TCPS, and researchers are compelled to stake their argument on article 3.7(c), that consent would be "impracticable". REBs differ widely on whether they will grant a waiver on that basis, depending on the specifics of the project. If you cannot obtain a waiver but believe that obtaining consent is impracticable, consider requesting a procedure whereby participants are informed about the observational research occurring (e.g., via signs, flyers) but their explicit consent is not required. Some REBs have approved manoeuvres like this. A final warning about waivers of consent: If your research involves data linkages between, for example, your research database and a provincial medical services or drug utilization database, find out in advance what the database owners demand in terms of consent. It is possible that your REB will grant a waiver but the database owner has a policy, based on its interpretation of provincial privacy legislation, requiring that data be supplied only where consent has been explicitly obtained.

A participant's signature on a consent form is the standard evidence of consent, but TCPS article 3.12 describes situations in which alternative means of demonstrating consent may be permissible. For example, participants may be physically unable to sign, or a participant's culture may discourage signing of documents because of perceived legal implications. If written consent is not obtained, the REB must first approve the alternate evidence of consent, and researchers are strongly advised to document what was explained to the participant, what questions the participants asked, and what evidence of consent was obtained.

The concept of informed consent is complex, involving ethical, legal, and cognitive dimensions. The fundamental tenets are that consent is voluntary (without undue influence or coercion, and can be withdrawn at any time), informed (disclosing "all information necessary for making an informed decision to participate in a research project" [TCPS2 article 3.2], of which there are many elements), and ongoing (researchers must continually inform participants of new information and must continually assess participants' willingness and ability to participate, including recognizing changes in their comprehension of the implications of their involvement). When withdrawing their consent, participants are also able to request withdrawal of their data from the study. Whether such data withdrawal is legal depends on whether the study is regulated by the Food and Drugs Act.

Despite researchers' best efforts, there is a body of evidence suggesting that after they have provided informed consent, research participants are frequently unable to recall important details about the research (including risks). ${ }^{13,14}$ A specific concern in randomized trials of therapeutic interventions is that participants sometimes participate because they are hoping to receive the experimental intervention or because participation represents an enhancement of their clinical care. This is called the "therapeutic misconception" of research participation. ${ }^{15}$

\section{Approaching Participants}

A frequently changing and highly variable ethical challenge concerns the "rules of engagement" for approaching potential research participants. Different REBs have different rules all aimed at achieving the same 2 basic aims: no contact by a researcher should be coercive, and nobody being contacted should be surprised or offended that the person contacting 
them knows their personal health information. Provincial privacy legislation, different in every province, tends not to address research specifically or extensively, but research is often affected by such legislation anyway. Institutional privacy officers are responsible to uphold that legislation on behalf of the institution, and they frequently make rules that are not intended for research but are applied to research nonetheless. This is an ever-changing palette, but a current example goes like this: Privacy legislation prohibits hospital clinics from giving lists of their patients to anyone without the patients' consent. Because there is no explicit exception for research, the institution declares that clinicians working in a clinic cannot use their own patient census as a basis for approaching potential research participants directly. Instead, the institution requires that a generic letter from the hospital be sent to all targeted patients asking them to explicitly consent to being contacted for research purposes by the clinic. Only patients who sign the form may be contacted, which drastically impedes enrolment. Learn your local rules of engagement and check frequently for changes.

\section{Paying Participants}

It is unethical to give inducements (i.e., money or any other form of non-health benefit) that could cause prospective participants to take on research risks that they otherwise would not accept, a process known as undue influence. The issues are complex, and this test falters when we consider that in phase 1 drug studies, participants take on risks with no prospect of benefit, often specifically for the money. The concept of free and informed consent supersedes the concern of undue influence in this case. Ultimately, REBs are required to judge whether the incentives being offered constitute undue influence, and this is not always easy.

\section{Registries}

Pharmacy practice researchers are becoming increasingly involved in creating patient registries for research purposes. The primary ethical issues here relate to what information is being collected and how it will be used and shared. The collection of data in registries generally involves minimal risk, but because of frequent publicized privacy breaches and because the specific research aims of registries are often necessarily vague, REBs pay a lot of attention to such projects. Consult your REB's registry guidelines if they exist. The primary aim is to clearly document exactly what is being collected from people, how it will be stored and for how long, what it will be linked with, and under what circumstances it will be shared and with whom, and to affirm that future projects based on the registry will be submitted for ethics review. The consent form should be exhaustive in all these regards.

\section{Consent Forms}

Researchers often pay relatively little attention to the consent form, whereas REBs tend to pay a lot of attention to it, which results in approval delays. REBs frequently review protocols that have been written by an experienced researcher in conjunction with a consent form that has been written by a student or research assistant barely familiar with the project. Most REBs have a consent form template. Use it. The REB looks unfavourably upon any language in the consent form that seems to encourage people to enrol. Instead, keep it simple and factual. Researchers frequently have difficulty stating the purpose of the research in lay language, so seek a layperson's help. Patients often see something like the following on a consent form: "This is a pilot study to ascertain whether a novel oscilloscopic $\mathrm{x}$-ray probe can differentiate premalignant from benign endothelial cells in the throat." A much improved lay explanation might be the following: "We are trying to find out if a new kind of $\mathrm{x}$-ray camera can tell us which cells in your throat might be becoming cancerous." A final tip regarding consent forms: Include only research-related procedures and risks in a consent form. Do not describe aspects of standard care, as doing so may lead to misinterpretation of standard care as part of the research.

\section{Banking Human Biological Materials}

TCPS article 2.1 defines "human biological materials" as "tissues, organs, blood, plasma, serum, DNA, RNA, proteins, cells, skin, hair, nail clippings, urine, saliva and other body fluids". Pharmacy practice researchers are sometimes involved in projects in which such materials are collected and retained for some future purpose, a process that invokes special ethical considerations. First, many REBs regard such activity as "tissue banking"; at a minimum, a detailed explanation of the storage conditions and location, access rules, confidentiality protections, rules for sharing the tissue, and potential uses will be required. Increasingly, institutions and REBs require the researcher to complete a special registration and certification program. Institutions are increasingly concerned about banks with reams of genetic material held by unknown people with vague stewardship and sharing arrangements. Registration and certification are designed to bring these banks "into the light". A final tip about banking biological materials: Researchers are allowed, with participants' consent, to collect human biological materials for unspecified future research purposes. REBs usually require that this be optional, i.e., not required for participation in the main study.

\section{SOURCES OF ETHICAL ADVICE}

This article has highlighted several ethical issues for pharmacy practice researchers to consider, but every project is 
different, and REBs judge research on a case-by-case basis. For help, try consulting the interpretations of the TCPS provided by the Canadian Interagency Panel on Research Ethics. ${ }^{16}$ Researchers can vastly reduce uncertainty and frustration by simply talking to the REB staff about their questions. They are there to help. Read the guidance notes provided by your REB, go to the seminars the REB offers, talk to people with experience. As you gain your own experience, share it with your colleagues.

\section{References}

1. Tri-Council policy statement: ethical conduct for research involving humans. 2nd ed. Ottawa (ON): Interagency Advisory Panel on Research Ethics; [updated 2011 Apr 7; cited 2014 Jan 18]. Available from: www.ethics.gc.ca/eng/ policy-politique/initiatives/tcps2-eptc2/Default/

2. The TCPS 2 tutorial course on research ethics (CORE). Ottawa (ON): Interagency Advisory Panel on Research Ethics; [updated 2013 Nov 12; cited 2014 Jan 18]. Available from: www.ethics.gc.ca/eng/education/tutorialdidacticiel/

3. Beecher HK. Ethics and clinical research. N Engl J Med. 1966;274(24):135460.

4. National Commission for the Protection of Human Subjects of Biomedical and Behavioral Research. The Belmont report: ethical principles and guidelines for the protection of human subjects of research. Washington (DC): US Department of Health, Education, and Welfare; 1979 [cited 2014 Jan 18]. Available from: www.hhs.gov/ohrp/humansubjects/guidance/belmont.html

5. Relman AS. Lessons from the Darsee affair. N Engl J Med 1983;308(23): 1415-7.

6. CFR - code of federal regulations title 21. Silver Springs (MD): US Food and Drug Administration; [updated 2013 Jun 1; cited 2014 Mar 13]. Available from: www.accessdata.fda.gov/scripts/cdrh/cfdocs/cffrr/cfrsearch.cfm

7. Institutional review boards frequently asked questions - information sheet. Silver Spring (MD): US Food and Drug Administration; [updated 2013 Aug 27; cited 2014 Jan 18]. Available from: www.fda.gov/regulatoryinformation/ guidances/ucm $126420 . \mathrm{htm}$

8. Canada’s strategy for patient-oriented research. Ottawa (ON): Canadian Institutes for Health Research; 2011 [cited 2014 Jan 18]. Available from: www.cihr-irsc.gc.ca/e/44000.html

9. BC ethics harmonization initiative. Vancouver (BC): Michael Smith Foundation for Health Research;[cited 2014 Jan 18]. Available from: www.msfhr.org/ our-work/activities/bc-ethics-harmonization-initiative

10. Clinical trial registration. In: Recommendations for the conduct, reporting, editing, and publication of scholarly work in medical journals. International Committee of Medical Journal Editors; [cited 2014 Mar 18]. Available from: www.icmje.org/recommendations/browse/publishing-and-editorial-issues/ clinical-trial-registration.html

11. Guidance document for clinical trial sponsors: clinical trial applications. Ottawa (ON): Health Canada; [updated 2013 Nov 19; cited 2014 Jan 18]. Available from: www.hc-sc.gc.ca/dhp-mps/prodpharma/applic-demande/guide-ld/clini/ ctdcta_ctddec-eng.php
12. Consultation TCPS 2 - proposed changes for public comment. Ottawa (ON): Interagency Advisory Panel on Research Ethics; [modified $2014 \mathrm{Feb} 4$; cited 2014 Mar 18]. Available from: www.pre.ethics.gc.ca/eng/english/ publicparticipation/callforcomments/consultation_20130912/

13. Akkad A, Jackson C, Kenyon S, Dixon-Woods M, Taub N, Habiba M. Patients' perceptions of written consent: questionnaire study. BMJ. 2006;333(7567):528.

14. Joffe S, Cook EF, Cleary PD, Clark JW, Weeks JC. Quality of informed consent in cancer clinical trials: a cross-sectional survey. Lancet. 2001; 358(9295):1772-7.

15. Townsend A, Cox SM. Accessing health services through the back door: a qualitative interview study investigating reasons why people participate in health research in Canada. BMC Med Ethics. 2013;14:40.

16. TCPS 2 interpretations. Ottawa (ON): Interagency Advisory Panel on Research Ethics; [updated 2013 Apr 19; cited 2014 Jan 18]. Available from: www.ethics.gc.ca/eng/policy-politique/interpretations/Default/

Peter Loewen, BSc(Pharm), ACPR, PharmD, FCSHP, RPh, is Director of the Doctor of Pharmacy Programs and Assistant Professor in the Faculty of Pharmaceutical Sciences, The University of British Columbia, and Pharmacotherapeutic Specialist (Medicine), Vancouver General Hospital, Vancouver, British Columbia. He is also Chair of the University of British Columbia Clinical Research Ethics Board.

Competing Interestes: None declared.

\section{Address correspondence to:}

Dr Peter Loewen

Faculty of Pharmaceutical Sciences

University of British Columbia

2405 Wesbrook Mall

Vancouver BC V6T $1 Z 3$

e-mail: peter.loewen@ubc.ca

This article is the third in the CJHP Research Primer Series, an initiative of the CJHP Editorial Board and the CSHP Research Committee. The planned 2-year series is intended to appeal to relatively inexperienced researchers, with the goal of building research capacity among practising pharmacists. The articles, presenting simple but rigorous guidance to encourage and support novice researchers, are being solicited from authors with appropriate expertise.

Previous articles in this series:

Bond CM. The research jigsaw: how to get started. Can J Hosp Pharm. 2014;67(1):28-30.

Tully MP. Research: articulting questions, generating hypotheses, and choosing study designs. Can J Hosp Pharm. 2014;67(1):31-4. 